\title{
Study of Electrical Conductivity and Microcosmic Structure of Tetrahedral Amorphous Carbon Films Doped by Boron
}

\author{
Xiaoyan Wang ${ }^{1,2}$ and Yuqing Zhao ${ }^{1}$ \\ ${ }^{1}$ Key Laboratory for Physical Electronics and Devices of the Ministry of Education, Xian Jiaotong University, Xi'an 710049, China \\ ${ }^{2}$ School of Electronics and Information Engineering, Taiyuan University of Science and Technology, Taiyuan 030021, China \\ Correspondence should be addressed to Xiaoyan Wang; 6882806@qq.com
}

Received 10 September 2015; Revised 8 December 2015; Accepted 10 December 2015

Academic Editor: Antonio Riveiro

Copyright (c) $2015 \mathrm{X}$. Wang and Y. Zhao. This is an open access article distributed under the Creative Commons Attribution License, which permits unrestricted use, distribution, and reproduction in any medium, provided the original work is properly cited.

\begin{abstract}
A type of tetrahedral amorphous carbon (ta-C) film that was doped by boron (ta-C:B) is focused on in this paper. The ta-C film is prepared by filtered cathodic vacuum arc (FCVA) technique and then doped with boron using the thermal diffusion method. Then the microcosmic structure and electrical conductivity of the ta- $\mathrm{C}$ are characterized by an $\mathrm{X}$-ray photoelectron spectroscopy (XPS) method and four-probe method, respectively. The results show that the conductivity of ta-C:B is significantly increased; the resistivity decreases from $1.5 \times 10^{6} \Omega \cdot \mathrm{cm}$ to $350 \Omega \cdot \mathrm{cm}$ approximately, while the percentage of $\mathrm{sp}^{3}$ bonds in the film is changed from $87 \%$ to $60 \%$ approximately. It means that this type of film preserved the mechanical characteristics of diamond-like carbon (DLC) films and improved the electrical characteristics greatly at the same time.
\end{abstract}

\section{Introduction}

Tetrahedral amorphous carbon (ta-C) film is a predominately $\mathrm{sp}^{3}$-bonded semiconductor with a band gap of order $2 \mathrm{eV}$ [13]. ta-C films have attracted considerable interest due to their unique mechanical, structural, and morphological properties $[4,5]$. The ta-C films are also being used in electronics, optoelectronics, vacuum microelectronics, microelectromechanical systems, and sensors [6-9]. Furthermore, latest research evidenced that the ta-C film can be used to achieve different surface functions and coatings for anticorrosion and electrochemical application [10].

The prospects for using ta-C as a semiconductor in electronic applications have been greatly enhanced with the discovery of the ta-C analogue to a-Si and a-Ge [11]. Ntype doping of ta-C has been observed when nitrogen is incorporated into the films and thin film transistors have been produced. P-type of ta-C films using boron incorporation continues to be an area of great interest and relevance to establish ta- $\mathrm{C}$ as a viable material for electronic application. Results published to date in the literature by several researchers with respect to both preparation and properties have been inconclusive. There are larger differences for the reported electrical properties of the film in results. For example, a report obtained from Chhowalla et al. Liverpool University has a reduction in conductivity of two orders of magnitude compared to undoped films [12]. Another team of Kleinsorge et al. from Cambridge University obtained excellent conductivity increases of five orders of magnitude [13], while the researches by Panwar and Han showed the conductivity increases of only one order of magnitude, respectively $[14,15]$.

As to the preparation and performance testing of ta$\mathrm{C}$ and ta-C:B films, there are several different techniques. Panwar et al. prepared boron- and phosphorus-incorporated tetrahedral amorphous carbon films deposited by the filtered cathodic vacuum arc process and tested the crystal growth, surfaces, interfaces, thin films, and bulk materials of the films [16]. And they deposited ta-C films by the S-bend filtered cathodic vacuum arc (FCVA) process, and the influences of substrate bias during growth and of hydrogen and nitrogen incorporation on density of states and field emission threshold were reported. And the best properties measured within the range of hydrogen and nitrogen incorporation [17] were presented. They also grew and hydrogenated tetrahedral amorphous carbon (ta-C, ta-C:H) films deposited by S-bend 
FCVA technique. It has been found that the hydrogenation of ta-C film improved the nanomechanical properties [18]. Tripathi et al. reported the effect of substrate bias on the structural, nanomechanical, field emission, and ammonia gas sensing properties of nitrogenated amorphous carbon films embedded with nanocrystallites (a-C:N:nc) deposited by a filtered anodic jet carbon arc (FAJCA) technique. The films are characterized by X-ray diffraction, high resolution transmission electron microscopy, energy dispersive X-ray spectroscopic analysis, Raman spectroscopy, nanoindentation, field emission, and ammonia gas sensing measurements. The properties of the films obtained are found to depend on the substrate bias [19]. They also doped hydrogenated amorphous silicon carbide ( $\mathrm{P}$ doped a-Si C:H) thin films deposited at room temperature by a FCVA technique using a phosphorus doped solid silicon target as a cathode in the presence of acetylene gas. These films have been characterized by X-ray diffraction (XRD), scanning electron microscopy, energy dispersive X-ray analysis, dark conductivity, activation energy, optical band gap, secondary ion mass spectroscopy, Raman spectroscopy, current-voltage, capacitancevoltage, and photoconductive measurements [20].

In this paper, a different method is adopted to obtain the boronated tetrahedral amorphous carbon (ta-C:B) film. Firstly, the ta- $C$ film is prepared by the FCVA technique; then the prepared film is boronated by the thermal diffusion technique. The microcosmic structure and conduction properties of the ta-C:B film were then tested and analyzed.

This paper is structured as follows. Section 2 describes the experiments of sample preparation and testing. The experimental results are discussed in Section 3. Finally, conclusion is given in Section 4.

\section{Experiment}

The experiment present in this paper contains the samples preparation and their measurements.

2.1. Sample Preparation. ta-C film is prepared using FCVA equipment. The cathodic material is the graphite with purity 99.99\%. The monocrystal silicon wafer with single face polished is used as the substrate. The silicon wafer is ultrasonically cleaned with absolute ethanol and acetone for $15 \mathrm{~min}$, respectively. The pressure of vacuum chamber is controlled at about $6 \times 10^{-4} \mathrm{~Pa}$; the voltage and frequency of the magnetic scanning coils are $100 \mathrm{v}$ and $50 \mathrm{HZ}$, respectively. The applied bias of substrate is $200 \mathrm{~V}$. The arcing voltage is $25 \mathrm{~V}$, and the arcing current is about $80 \mathrm{~A}$. To ensure the uniformity of taC coatings, the sample rotation is with the speed of $3 \mathrm{r} / \mathrm{min}$. Five samples are prepared with the deposition time of $30 \mathrm{~min}$.

As to the preparation of ta-C:B, the thermal diffusion method is used. The samples are cleaned with absolute ethanol and acetone for $15 \mathrm{~min}$, respectively. Boron nitride was chosen as the diffusion material. Dry boron nitride crystal powder was evenly placed on the diffusion material carrier with nitrogen as the environment protection gas, and the flux was maintained at $500 \mathrm{~mL} / \mathrm{min}$. The temperature was then raised to $960^{\circ} \mathrm{C}$ by heating, and the fabricated sample is placed in the diffusion chamber. The samples are numbered: number 1 is untreated by thermal diffusion and number 2 , number 3 , number 4 , and number 5 are diffused with $5 \mathrm{~min}$, $10 \mathrm{~min}, 15 \mathrm{~min}$, and $20 \mathrm{~min}$, respectively. Once the diffusion was complete, all samples were cooled to room temperature in a nitrogen environment.

2.2. Measurements of the Samples. Two main properties of the ta-C:B samples are tested in this study, microcosmic structure and electrical conductivity. The Thermo K-Alpha high performance X-ray photoelectron spectroscopy imager was used to analyze the energy spectrum of the ta-C:B film to analyze its microcosmic structure. As to the testing of the electrical conductivity, the RTS-8 four-probe tester is used. At a constant temperature, seven areas from each sample were chosen to test the film's resistivity characteristic.

\section{Results and Discussion}

3.1. Structural Analysis of ta-C:B Film. The Thermo K-Alpha $\mathrm{X}$-ray photoelectron spectroscopy imager was used to analyze the energy spectrum of the ta-C:B film. The Bls spectrogram is shown in Figure 1, which shows that the B1s spectral peaks of boron in ta-C:B film all clearly approach $189 \mathrm{eV}$.

The C1s core energy spectrogram obtained using the $\mathrm{X}$-ray photoelectronic spectroscopy imager with different thermal diffusion times is shown in Figure 2. From Figure 2, it can be clearly seen that when boron is not diffused into the ta-C film, C1s spectral peak is closer to $285.1 \mathrm{eV}$, indicating a higher number of $\mathrm{sp}^{3}$ bonds within the film, which can be calculated as approximately $87 \%$ from the Gaussian peak area ratio. Figures 2 (a) to 2 (c) show that when the diffusion time of boron increases, C1s' spectral peaks gradually move towards $284.2 \mathrm{eV}$, which is the spectral peak of the graphite phase. The longer the diffusion time is, the greater the spectral peak intensity of $\mathrm{sp}^{2}$ is; meanwhile the $\mathrm{sp}^{3}$ spectral peak intensity gradually decreases. This indicates that the ratio of $\mathrm{sp}^{3} / \mathrm{sp}^{2}$ bonds within the film is decreasing, due to the bonding method of some of the carbon atoms changing from $\mathrm{sp}^{3}$ to $\mathrm{sp}^{2}$, causing the film to begin to exhibit signs of graphitization, while the carbon atoms in the ta-C:B films are mainly in $\mathrm{sp}^{3}$ hybridization which tend to decrease as boron content increases.

From Figures 2(c), 2(d) and 2(e), it can be observed that as the thermal diffusion time increases, the spectral peak of $283.1 \mathrm{eV}$ of the boron-carbon bonds becomes gradually more evident and the intensity increases, which indicates that more $\mathrm{C}$ and $\mathrm{B}$ bonds are forming boron-carbon compounds. During this stage, the ratio of $\mathrm{sp}^{2}$ bonds and $\mathrm{sp}^{3}$ bonds remains basically unchanged, illustrating that the graphitization tendency of ta-C film is inhibited. Meanwhile the number of $\mathrm{sp}^{3}$ bonds within the film remains above $60 \%$ throughout the experiment, indicating that the film still adequately maintains the fine qualities of diamond-like carbon films.

According to the analyzing of the $\mathrm{Cls}$ and B1s core energy spectrogram, it is clear that the percentage of $\mathrm{sp}^{3}$ bond 


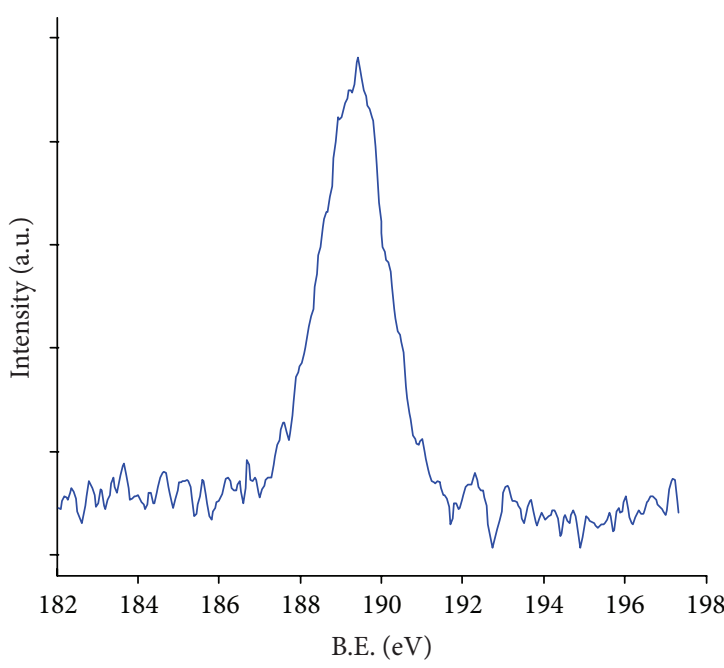

(a) Thermal diffusion doping 5 min

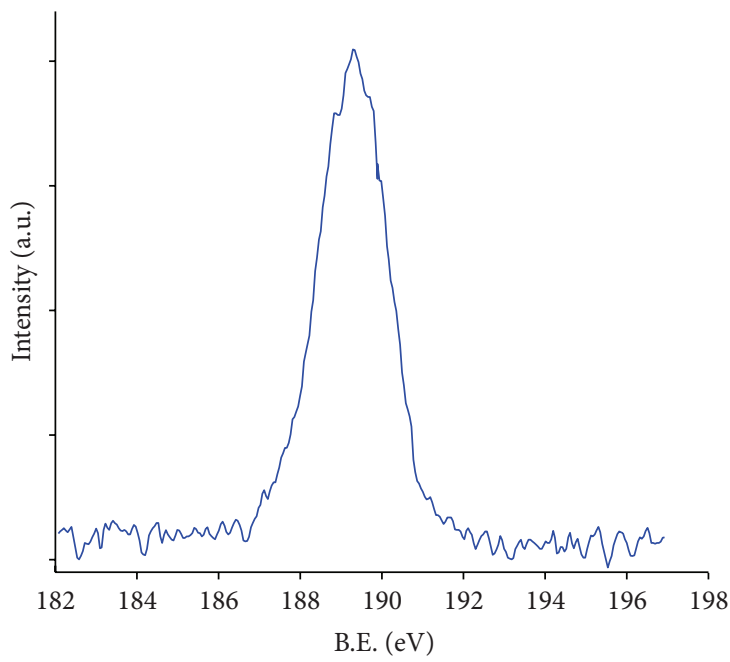

(c) Thermal diffusion doping $15 \mathrm{~min}$

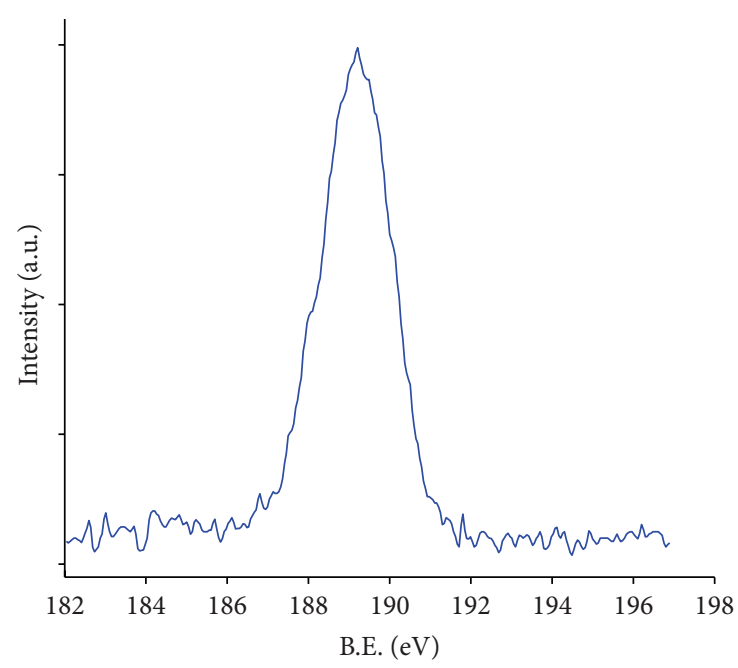

(b) Thermal diffusion doping $10 \mathrm{~min}$

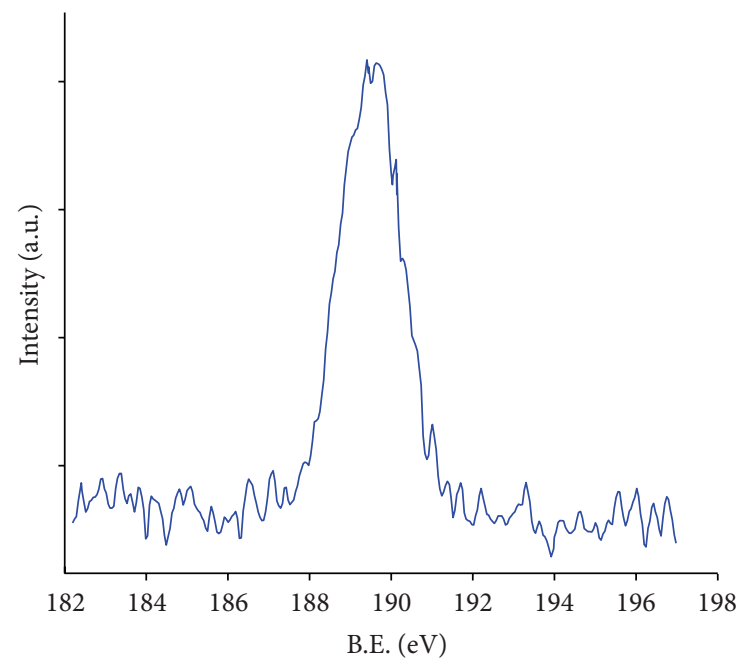

(d) Thermal diffusion doping $20 \mathrm{~min}$

FIgURE 1: Fitting curve of B1s core level spectrogram of ta-C:B film with different thermal diffusion times.

reduces while $\mathrm{sp}^{2}$ bond increases for the doping of the boron atom by thermal diffusion method. The major bonding mode of $\mathrm{sp}^{2}$ is that the boron atom replaces the carbon atom in the graphite. When a $\mathrm{sp}^{2}$ position is replaced by a boron atom, the BC3 structure will be formed [21]. This process can lose the framework of the original structure. According to the theory of shallow implantation, this process can restrain the formation of the $\mathrm{sp}^{3}$ bond; therefore, the percentage of $\mathrm{sp}^{3}$ bond reduces. As a result, a BC6 structure will be formed surrounding the boron atom as in Figure 3 , which is an available doping way to enhance the electrical conductance. When the diffusion time is short, there are few boron atoms doped in the ta-C film, and the doped boron and carbon atoms only combine around the interface of the film, which results in a low percentage of $\mathrm{sp}^{3}$ bond. Along with the increasing of the diffusion time, more and more boron atoms take the $\mathrm{sp}^{2}$ position, which increases the percentage of $\mathrm{B}-\mathrm{C}$ $\mathrm{sp}^{2}$ bond. In the next stage, the content of the boron atom in the film will rise continuously. A type of B-C compound will be formed to accommodate more boron atoms. This process restrains the formation of the graphitizing of the film, and the percentage of $\mathrm{sp}^{2}$ and $\mathrm{sp}^{3}$ will be constant approximately.

3.2. Analysis of Conducting Property of ta-C:B. The conduction properties of sample films were tested with an RTS-8 four-probe tester. The resistivity of each sample is shown in Table 1. At a constant temperature, seven areas from each sample were chosen to test the film's resistivity characteristic, and the results are shown in Figure 4. From this figure, it can be observed that as diffusion time increases, the overall resistivity of the ta-C:B film exhibits a trend of firstly decreasing and then increasing. At room temperature, the resistivity of ta-C film undoped with boron is about $1.5 \times$ $10^{6} \Omega \cdot \mathrm{cm}$; after diffusion with boron for 5 minutes, the 


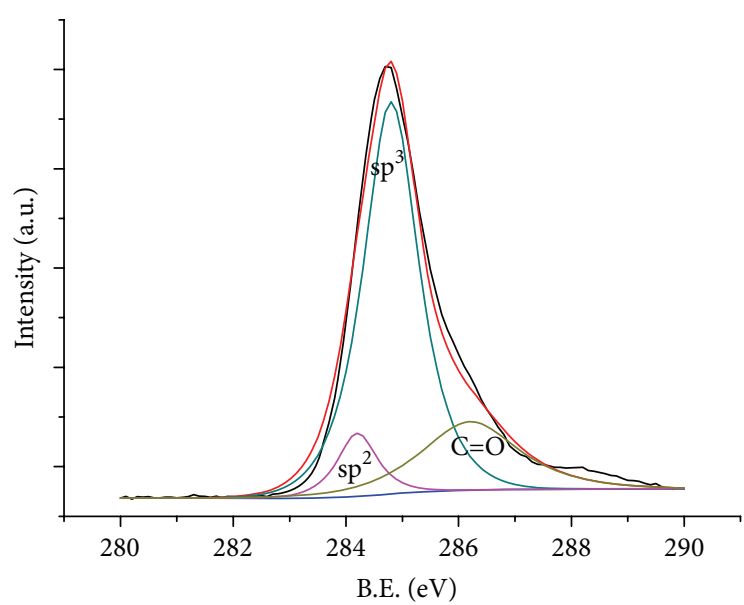

(a) ta-C film without doping

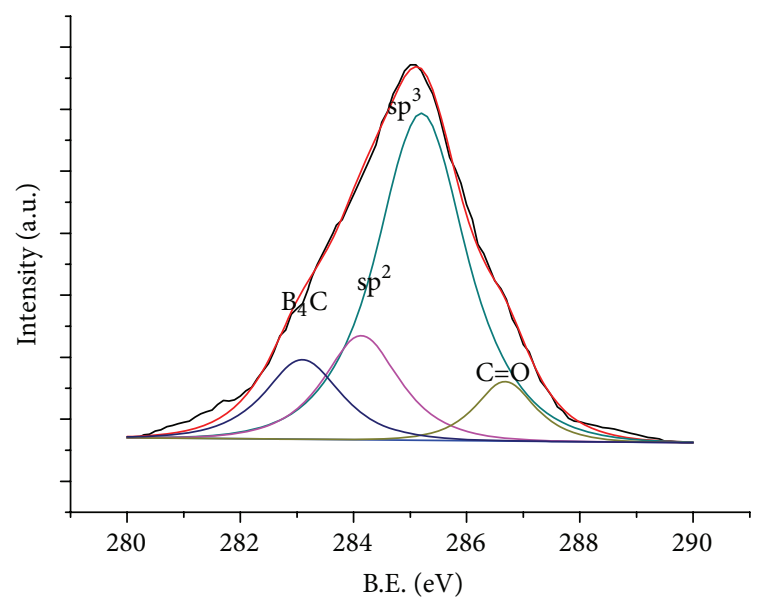

(c) ta-C:B film with thermal diffusion doping $10 \mathrm{~min}$

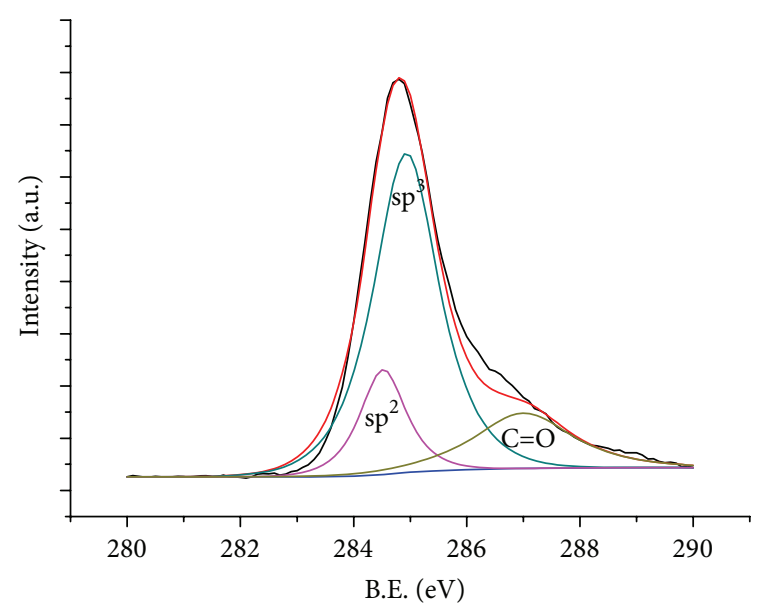

(b) ta-C:B film with thermal diffusion doping $5 \mathrm{~min}$

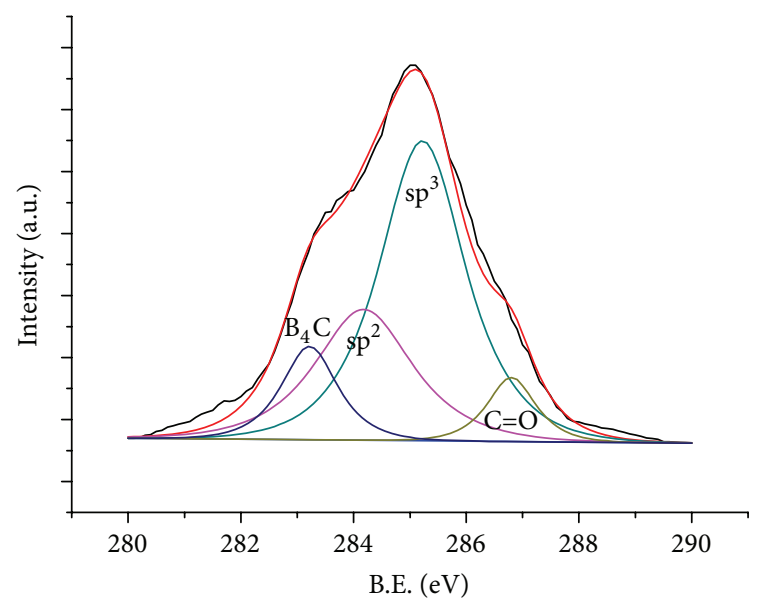

(d) ta-C:B film with thermal diffusion doping $15 \mathrm{~min}$

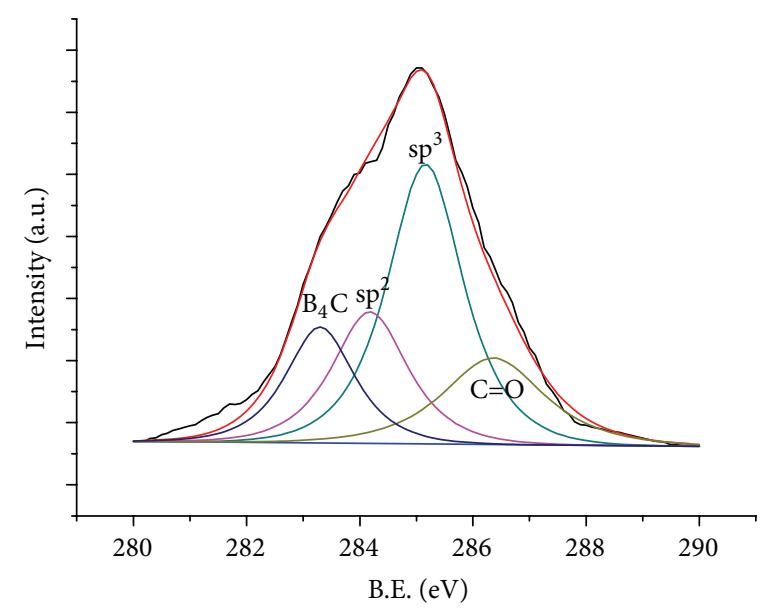

(e) ta-C:B film with thermal diffusion doping $20 \mathrm{~min}$

Figure 2: Fitting curve of C1s energy level spectrogram of ta-C film before and after boron doping.

resistivity exhibits evident reduction, to about $350 \Omega \cdot \mathrm{cm}$. When the diffusion time is increased to 10 minutes, the film's resistivity continues to decrease, although the actual decrease is small, only $250 \Omega \cdot \mathrm{cm}$. When the diffusion time is further increased to 15 minutes, the resistivity of ta-C:B film exhibits a rebound and experiences a large increase compared with diffusion times of 5 minutes and 10 minutes, to approximately $4100 \Omega \cdot \mathrm{cm}$. Thereafter, as the diffusion time 
TABLE 1: Test results of samples' resistivity.

\begin{tabular}{|c|c|c|c|c|c|c|c|c|}
\hline Test point & 1 & 2 & 3 & 4 & 5 & 6 & 7 & Average \\
\hline $\begin{array}{l}\text { Resistivity of ta-C film without doping } \\
(\Omega \cdot \mathrm{cm})\end{array}$ & $1.989 E 6$ & $1.989 E 6$ & $1.514 E 6$ & $1.051 E 6$ & $1.036 E 6$ & $1.338 E 6$ & $1.506 E 6$ & $1.489 E 6$ \\
\hline $\begin{array}{l}\text { Resistivity of ta-C:B film with thermal } \\
\text { diffusion doping } 5 \min (\Omega \cdot \mathrm{cm})\end{array}$ & 326 & 326 & 327 & 411 & 376 & 349 & 402 & 359 \\
\hline $\begin{array}{l}\text { Resistivity of ta-C:B film with thermal } \\
\text { diffusion doping } 10 \mathrm{~min}(\Omega \cdot \mathrm{cm})\end{array}$ & 252 & 253 & 254 & 259 & 264 & 258 & 213 & 250 \\
\hline $\begin{array}{l}\text { Resistivity of ta-C:B film with thermal } \\
\text { diffusion doping } 15 \min (\Omega \cdot \mathrm{cm})\end{array}$ & 4160 & 4101 & 4342 & 4520 & 4226 & 4220 & 4461 & 4287 \\
\hline $\begin{array}{l}\text { Resistivity of ta-C:B film with thermal } \\
\text { diffusion doping } 20 \mathrm{~min}(\Omega \cdot \mathrm{cm})\end{array}$ & 4459 & 4552 & 4401 & 3854 & 4047 & 4266 & 4979 & 4365 \\
\hline
\end{tabular}<smiles>CC(C)=C(C)B(C(C)=C(C)C)C(C)=C(C)C</smiles>

Figure 3: $\mathrm{B}-\mathrm{C} \mathrm{sp}{ }^{2}$ bond in the $\mathrm{BC}_{6}$ structure of ta-C:B.

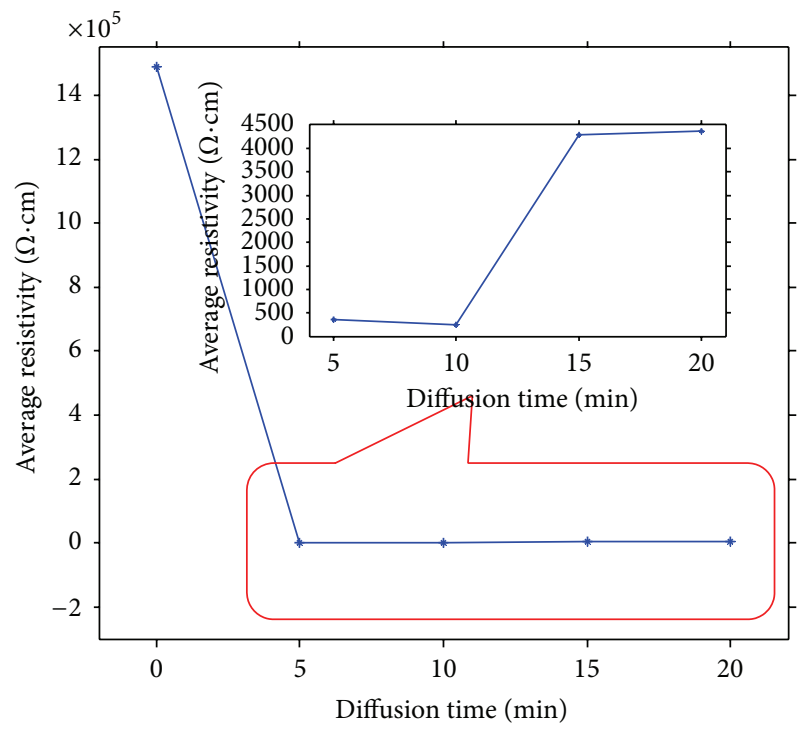

Figure 4: Average resistivity at different diffusion time.

is increased, the relative number of boron atoms within the film continues to increase, and the resistivity also increases slightly, to approximately $5000 \Omega \cdot \mathrm{cm}$.

Section 3.1 shows that there are three steps of boron in the ta-C:B film. One is the formation of the B-C $s p^{2}$ bond which can reduce the $\mathrm{sp}^{3}$ bond and produce a trend of graphitizing of the film. Another is the B-C compound which can increase the resistivity of the film. Combining the electrical conduction testing results, the following is clear. When the diffusion time is $5 \mathrm{~min}$, the bonding method in the ta-C:B film is the $\mathrm{B}-\mathrm{C} \mathrm{sp}^{2}$ bond, which has a lower resistivity; when the diffusion time is $10 \mathrm{~min}$, the content of boron atom still increases in the film, but some of it is the $\mathrm{B}-\mathrm{C}$ compound, so the resistivity of the film decreases but the magnitude is very small; when the diffusion time rises, the percentage of B$\mathrm{C}$ compound in the doping boron in the film increases, which can restrain the graphitizing and increase the resistivity at the same time.

\section{Conclusion}

In this work, we studied the microcosmic structure and electrical conductivity property of boron doped tetrahedral amorphous carbon (ta-C:B) film, which was doped using the thermal diffusion doping technique. It was discovered that when the number of $\mathrm{sp}^{3}$ bonds of ta-C:B film remains above $60 \%$, the film still preserves the characteristics of diamond-like carbon films. While the electrical conductivity is significantly increased, the resistivity decreases from $1.5 \times$ $10^{6} \Omega \cdot \mathrm{cm}$ to about $350 \Omega \cdot \mathrm{cm}$. Due to this fact, ta-C:B films could become a potential candidate for eventual use in the microelectronic application.

\section{Conflict of Interests}

The authors declare that there is no conflict of interests regarding the publication of this paper.

\section{Acknowledgments}

This research is supported by the National Natural Science Foundation of China (11405125), China Postdoctoral Science Foundation Fund Project (2014M562420), and the Fundamental Research Funds for the Central Universities.

\section{References}

[1] P. J. Martin, S. W. Filipczuk, R. P. Netterfield, J. S. Field, D. F. Whitnall, and D. R. McKenzie, "Structure and hardness of diamond-like carbon films prepared by arc evaporation," Journal of Materials Science Letters, vol. 7, no. 4, pp. 410-412, 1988.

[2] J. Robertson, "Properties of diamond-like carbon," Surface and Coatings Technology, vol. 50, no. 3, pp. 185-203, 1992. 
[3] C. W. Chen and J. Robertson, "Doping mechanism in tetrahedral amorphous carbon," Carbon, vol. 37, no. 5, pp. 839-842, 1999.

[4] B. Bhushan and V. N. Koinkar, "Microscale mechanical and tribological characterization of hard amorphous carbon coatings as thin as $5 \mathrm{~nm}$ for magnetic disks," Surface and Coatings Technology, vol. 76-77, no. 2, pp. 655-669, 1995.

[5] K. S. Lim, "Boron-doped amorphous diamondlike carbon as a new p-type window material in amorphous silicon p-i-n solar cells," Applied Physics Letters, vol. 72, no. 1, pp. 106-108, 1998.

[6] B. S. Satyanarayana, J. Robertson, and W. I. Milne, "Low threshold field emission from nanoclustered carbon grown by cathodic arc," Journal of Applied Physics, vol. 87, no. 6, pp. 31263131, 2000.

[7] F. J. Clough, W. I. Milne, B. Kleinsorge, J. Robertson, G. A. J. Amaratunga, and B. N. Roy, "Tetrahedrally bonded amorphous carbon (ta-C) thin film transistors," Electronics Letters, vol. 32, no. 5, pp. 498-499, 1996.

[8] R. Kalish, Y. Lifshitz, K. Nugent, and S. Prawer, "Thermal stability and relaxation in diamond-like-carbon. A Raman study of films with different $\mathrm{sp}^{3}$ fractions (ta-C to a-C)," Applied Physics Letters, vol. 74, no. 20, pp. 2936-2938, 1999.

[9] J. T. H. Tsai, K. B. K. Teo, and W. I. Milne, "Approach for a self-assembled thin film edge field emitter," Journal of Vacuum Science \& Technology B, vol. 20, no. 1, pp. 1-4, 2002.

[10] S. Neuville, "New application perspective for tetrahedral amorphous carbon coatings," QScience Connect, vol. 8, 2014.

[11] D. R. McKenzie, D. Muller, and B. A. Pailthorpe, "Compressivestress-induced formation of thin-film tetrahedral amorphous carbon," Physical Review Letters, vol. 67, no. 6, pp. 773-776, 1991.

[12] M. Chhowalla, Y. Yin, G. A. J. Amaratunga, D. R. McKenzie, and T. Frauenheim, "Highly tetrahedral amorphous carbon films with low stress," Applied Physics Letters, vol. 69, no. 16, pp. 23442346, 1996.

[13] B. Kleinsorge, A. Ilie, M. Chhowalla, W. Fukarek, W. I. Milne, and J. Robertson, "Electrical and optical properties of boronated tetrahedrally bonded amorphous carbon (ta-C:B)," Diamond and Related Materials, vol. 7, no. 2-5, pp. 472-476, 1998.

[14] O. S. Panwar, M. A. Khan, B. S. Satyanarayana, S. Kumar, and Ishpal, "Properties of boron and phosphorous incorporated tetrahedral amorphous carbon films grown using filtered cathodic vacuum arc process," Applied Surface Science, vol. 256, no. 13, pp. 4383-4390, 2010.

[15] J. Han, M. Tan, J. Zhu et al., "Photovoltaic characteristics of amorphous silicon solar cells using boron doped tetrahedral amorphous carbon films as p-type window materials," Applied Physics Letters, vol. 90, no. 8, Article ID 083508, 2007.

[16] O. S. Panwar, M. A. Khan, M. Kumar et al., "Characterization of boron- and phosphorous-incorporated tetrahedral amorphous carbon films deposited by the filtered cathodic vacuum arc process," Japanese Journal of Applied Physics, vol. 48, no. 6, Article ID 065501, 2009.

[17] O. S. Panwar, M. A. Khan, B. S. Satyanarayana et al., "Effect of high substrate bias and hydrogen and nitrogen incorporation on density of states and field-emission threshold in tetrahedral amorphous carbon films," Journal of Vacuum Science \& Technology B, vol. 28, no. 2, pp. 411-422, 2010.

[18] O. S. Panwar, R. K. Tripathi, and S. Chockalingam, "Improved nanomechanical properties of hydrogenated tetrahedral amorphous carbon films measured with ultra low indentation load," Materials Express, vol. 5, no. 5, pp. 410-418, 2015.
[19] R. K. Tripathi, O. S. Panwar, A. K. Srivastava, I. Rawal, and S. Chockalingam, "Structural, nanomechanical, field emission and ammonia gas sensing properties of nitrogenated amorphous carbon films deposited by filtered anodic jet carbon arc technique," Talanta, vol. 125, no. 1, pp. 276-283, 2014.

[20] R. K. Tripathi, O. S. Panwar, A. K. Kesarwani et al., "Investigations on phosphorous doped hydrogenated amorphous silicon carbide thin films deposited by a filtered cathodic vacuum arc technique for photo detecting applications," RSC Advances, vol. 4, no. 97, pp. 54388-54397, 2014.

[21] E. C. Onyiriuka, "Aluminum, titanium boride, and nitride films sputter-deposited from multicomponent alloy targets studied by XPS," Applied Spectroscopy, vol. 47, no. 1, pp. 35-37, 1993. 

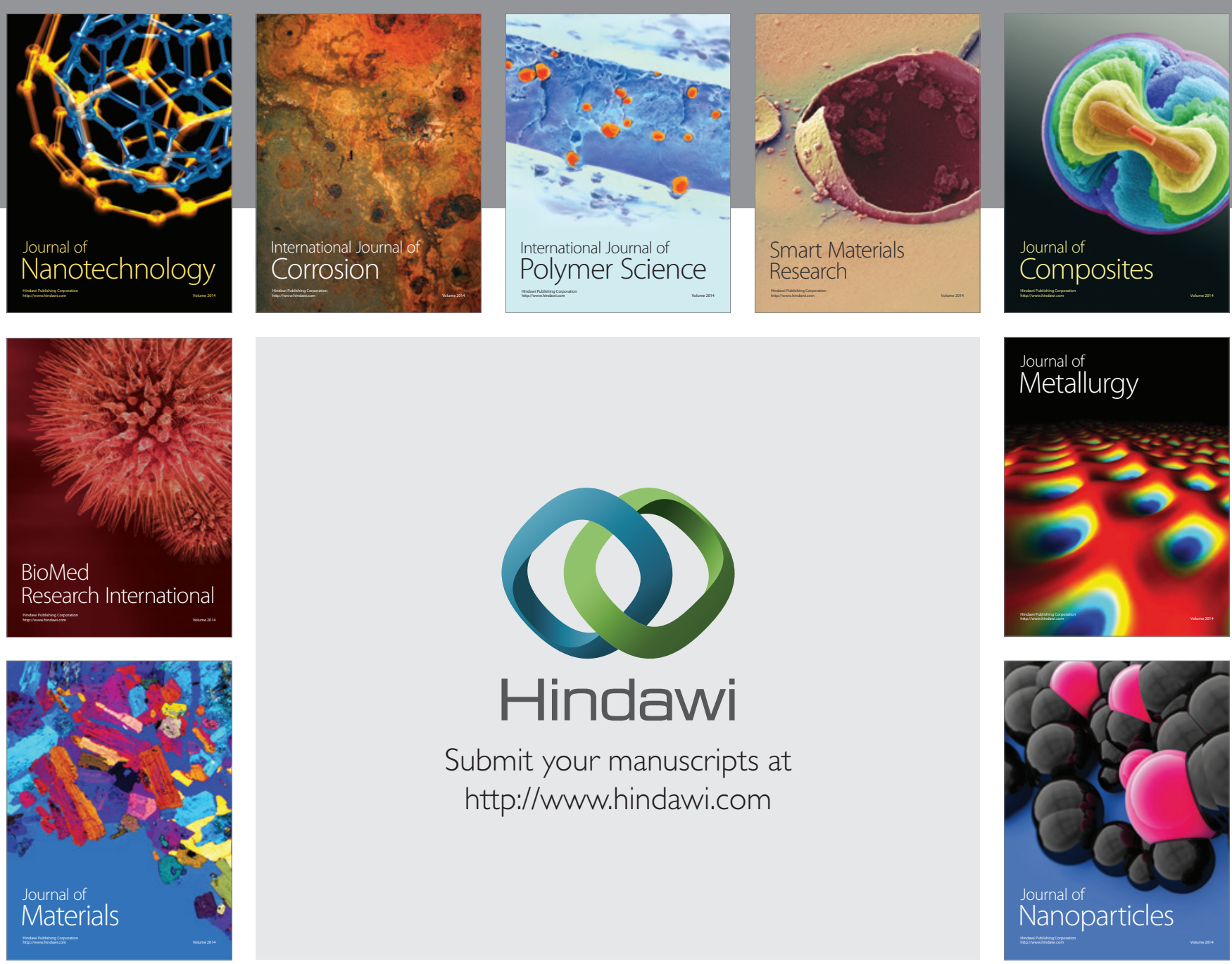

Submit your manuscripts at http://www.hindawi.com
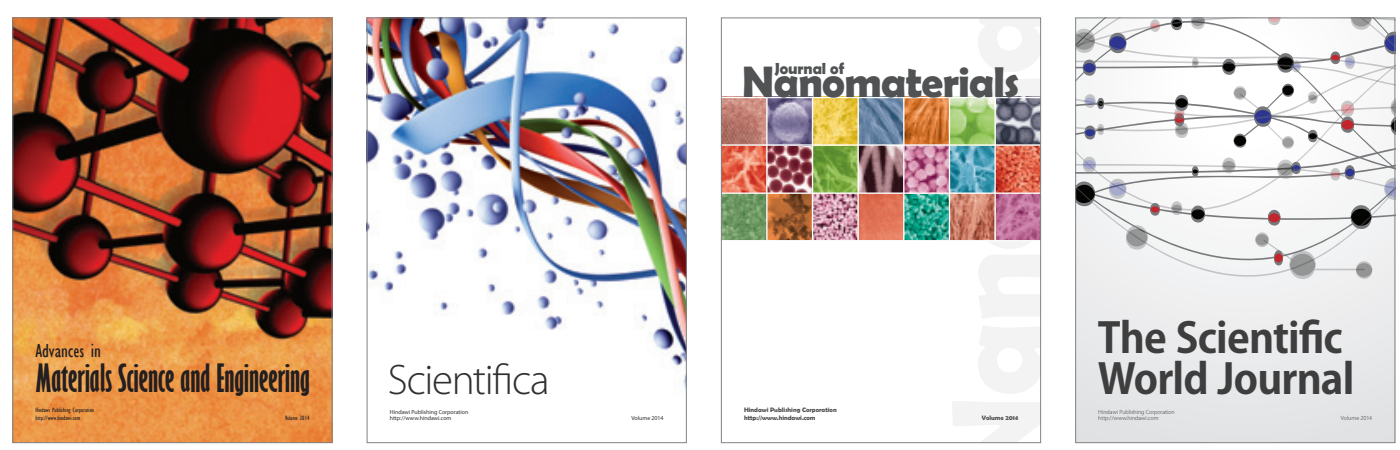

\section{The Scientific World Journal}
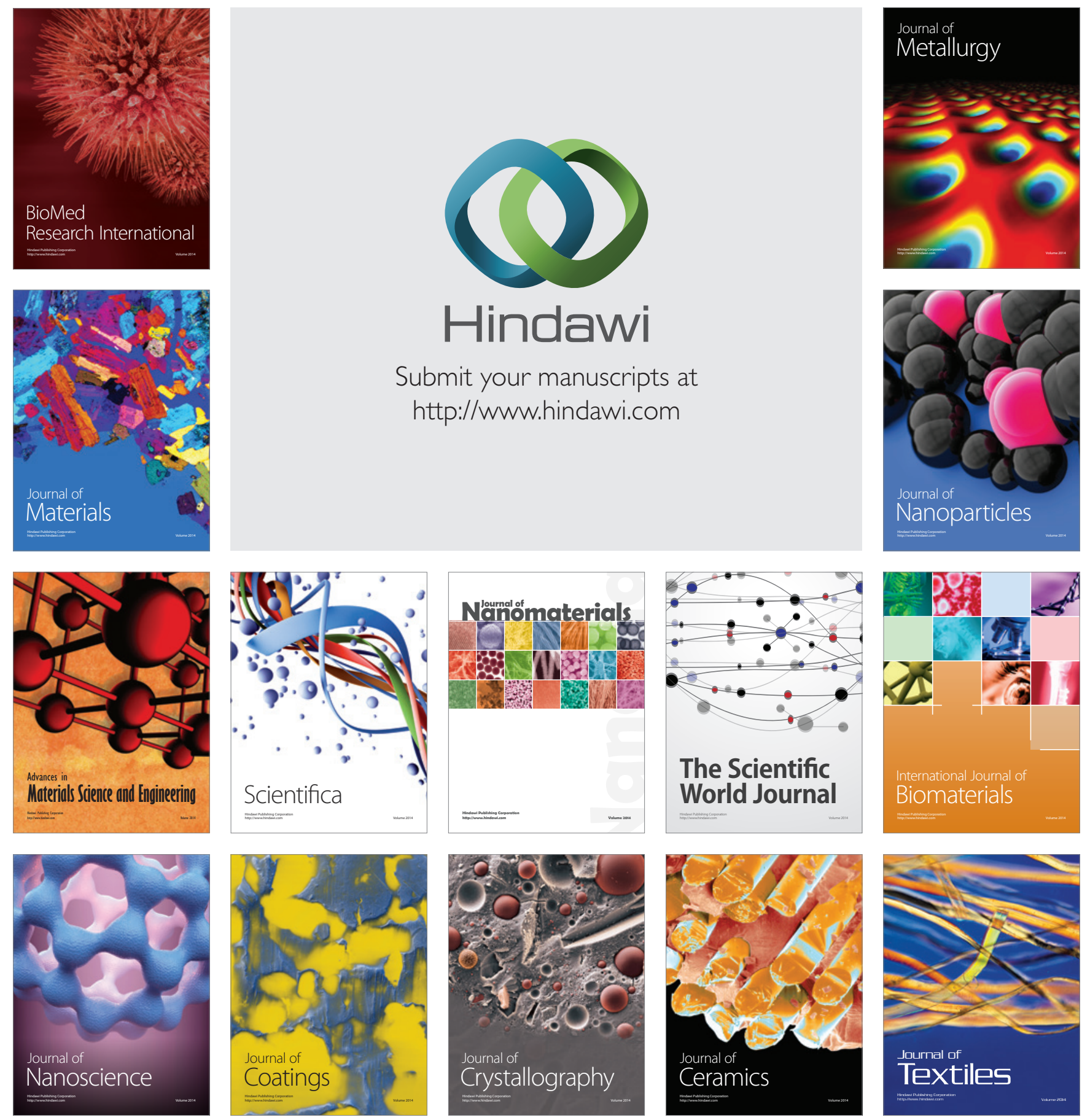